\title{
Understanding primate brain evolution
}

\author{
R. I. M. Dunbar* and Susanne Shultz \\ British Academy Centenary Research Project, School of Biological Sciences, University of Liverpool, \\ Liverpool L69 7ZB, UK
}

\begin{abstract}
We present a detailed reanalysis of the comparative brain data for primates, and develop a model using path analysis that seeks to present the coevolution of primate brain (neocortex) and sociality within a broader ecological and life-history framework. We show that body size, basal metabolic rate and life history act as constraints on brain evolution and through this influence the coevolution of neocortex size and group size. However, they do not determine either of these variables, which appear to be locked in a tight coevolutionary system. We show that, within primates, this relationship is specific to the neocortex. Nonetheless, there are important constraints on brain evolution; we use path analysis to show that, in order to evolve a large neocortex, a species must first evolve a large brain to support that neocortex and this in turn requires adjustments in diet (to provide the energy needed) and life history (to allow sufficient time both for brain growth and for 'software' programming). We review a wider literature demonstrating a tight coevolutionary relationship between brain size and sociality in a range of mammalian taxa, but emphasize that the social brain hypothesis is not about the relationship between brain/neocortex size and group size per se; rather, it is about social complexity and we adduce evidence to support this. Finally, we consider the wider issue of how mammalian (and primate) brains evolve in order to localize the social effects.
\end{abstract}

Keywords: brain evolution; life history; neocortex; primate; social brain hypothesis

\section{INTRODUCTION}

The social brain hypothesis was originally proposed explicitly as an explanation for the fact that primates have unusually large brains for body size when compared with other vertebrates (including all other mammals; Byrne \& Whiten 1988; Barton \& Dunbar 1997; Dunbar 1998). Its main claim was that because they had an unusually complex social life, primates needed a comparably large brain to cope with the computations involved. Initially, it was assumed that the social brain hypothesis applied only to primates. However, a number of analyses subsequently suggested that the hypothesis might apply more widely to other mammalian groups (including whales, carnivores and at least some insectivores; Marino 1996; Dunbar \& Bever 1998). However, these studies were limited in scope and focused only on possible correlations between sociality and brain size.

Two issues arise out of these findings. One fact is that, with the exception of Shultz \& Dunbar (2006), the focus has been mainly on bivariate correlations between brain size and either sociality (usually indexed as social group size) or some ecological variable (e.g. range size, an index of frugivory); little attempt has been made to evaluate whether the relationship between group size and brain size is actually a confound of these ecological variables. In part, this reflected a methodological constraint; existing statistical tools made it difficult to

\footnotetext{
* Author for correspondence (rimd@liv.ac.uk).
}

Electronic supplementary material is available at http://dx.doi.org/10. 1098/rstb.2006.2001 or via http://www.journals.royalsoc.ac.uk.

One contribution of 19 to a Dicussion Meeting Issue 'Social intelligence: from brain to culture'. evaluate dichotomous and quantitative variables in the same model. Statistical methods that have been developed recently now make it possible both to test a wider range of hypotheses simultaneously and, more importantly perhaps, to include dichotomous as well as continuous variables in the same analysis. They also allow the problem of phylogenetic inertia to be handled in a more sophisticated way than was previously the case. The second issue raised by these findings is the question as to just what it is about sociality that creates the cognitive load that is so demanding of neural computational power. There has been a tendency for the social brain hypothesis to be couched solely in terms of group size (the variable that most analyses have used as their assay of sociality). But, in fact, the hypothesis has quite explicitly always been about the complexity of social relationships (their quality rather than merely their quantity). Group size is certainly a correlate of social complexity (if only because the number of dyads and triads that have to be tracked and managed socially increases as a power function of the number of individuals in the group), but it is at best a crude proxy.

We address these two issues here. First, we present new analyses which incorporate a number of additional ecological and demographic variables. Our aims here are: (i) to confirm that the original findings hold up across a wider mammalian perspective when new more powerful statistical methods are used and (ii) to view brain evolution within a broader ecological and lifehistory perspective. Second, we consider in more detail the nature of the socio-cognitive demands that underpin the social brain effects and the way in which these relate to brain structure.

First, however, it is necessary to clarify one point that seems to have been repeatedly confused in the 
literature. The social brain hypothesis has sometimes been interpreted as though it was ecology-free and represented a contrast between ecology and sociality as the driving force of brain evolution. This is possibly because, in the original analyses (Dunbar 1992), sociality was pitted against a set of alternative ecological hypotheses. It is important to remember that the social brain hypothesis is itself an ecological hypothesis; the claim is that one or more ecological problems (survival, foraging, rearing offspring) are more effectively solved socially than by an individual's unaided efforts. The key issue here is whether an animal's solutions to the problems of successful survival and reproduction are social (either as an emergent property of group living or, perhaps, through social imitation) or are the product of individual problem solving. In other words, is the relationship between brain size and ecology direct (e.g. via trialand-error learning or individual problem solving) or indirect (mediated via sociality)?

More importantly, a clear distinction can be drawn between two versions of the cognitive challenge that underpins the social intelligence hypothesis. These differ in terms of what they consider the critical factor selecting for differences in socio-cognitive abilities. One view (that encompassed in the original Machiavellian hypothesis of Byrne \& Whiten (1988), and developed in more detail by Dunbar $(1992,1998)$ ) focuses on social bonding of groups as the critical issue. The other assumes that feeding (and hence nutrient flow) is the critical constraint, and thus that social learning of efficient foraging strategies has been the principal selection pressure for the evolution of socio-cognitive skills (Reader \& Laland 2002). Both the primate and the general vertebrate literatures have remained ambivalent as to which of these has been the more important force of natural selection, although the consensus is that predation risk has been the more important influence on primate social evolution (Dunbar 1988).

\section{THE SOCIAL BRAIN IN MAMMALIAN PERSPECTIVE}

The social brain hypothesis has been extensively tested on primates (Sawaguchi \& Kudo 1990; Dunbar 1992, 1998; Barton 1993, 1996; Deaner et al. 2000) and the results seem, in general, to be robust with respect to statistical methodology. While there have been a number of analyses suggesting that the social brain hypothesis might also hold in, at least, some other nonprimate mammals (Marino 1996, 2004; Dunbar \& Bever 1998), there have been few detailed tests of the hypothesis for mammalian groups other than primates. However, such analyses have recently been published for both carnivores and ungulates. Perez-Barberia \& Gordon (2005) and Shultz \& Dunbar (2006) have independently shown that sociality correlates with both relative brain and relative neocortex size in ungulates (social species have bigger brains/neocortices). Group size and diet made no independent contribution, but there was a significant independent effect of preferred habitat type. In this case, habitat was considered as a trichotomy (open, closed and mixed) and species adapted to mixed habitats had larger brains than those that were open or closed habitat specialists.

More importantly, perhaps, Perez-Barberia et al. (submitted) have shown, using Pagel's Discrete method, that for all three orders, there is very strong coevolution between relative brain (or neocortex) size and sociality in these two orders, as well as in primates; the two traits tend to change in synchrony rather than sequentially within each order's phylogenetic tree. This implies that the two traits are under tightly coupled selection; changes in one trait (sociality) are only possible if they are associated with changes in the other trait (brain size). This result lends support to the social brain hypothesis and weakens the alternative hypothesis that sociality is a by-product of having a large brain, when large brains have evolved for some other more conventional reason (e.g. to solve some ecological problem on a non-social basis).

Nonetheless, there was evidence in the Perez-Barberia et al. (submitted) analyses for lagged evolution in all three orders (primates, ungulates and carnivores). In primates, there were back transitions from large-brain/ more-social to small-brain/more-social and forward transitions from large-brain/less-social to large-brain/ more-social, but there were no consistent pathways whereby primates moved from small-brain/less-social to large-brain/more-social via intermediate steps. In ungulates, there seemed to have been a certain amount of switching back and forth between large-brain/social and, on one hand, small-brain/social and large-brain/ asocial, but again without any suggestion that these constituted intermediate steps in an evolutionary sequence. Carnivores exhibited much greater flexibility, with most transitional steps occurring more often than expected despite the fact that, overall, they showed the same strong correlated evolution. This suggests that in a limited number of cases (but especially in carnivores), it has been possible to develop a degree of sociality without large brains and we would predict that, in these cases, sociality is characterized by some 'looseness' (i.e. lack of group cohesion). We might interpret this finding as implying that sociality can more easily be decoupled from brain (or cognitive) constraints in carnivores than is possible in either ungulates or primates.

\section{PRIMATE BRAIN EVOLUTION REVISITED}

In the light of these new findings, we have undertaken new analyses of the primate data, in order to be able to evaluate in greater depth the relationships between primate brain size and species-specific behavioural, ecological and life-history characteristics. A number of recent studies have sought to evaluate the relative importance of ecology (Reader \& Laland 2002), life history (Ross 1992; Joffe 1997; Deaner et al. 2003) and allometric scaling relationships (Finlay \& Darlington 1995; Finlay et al. 2001; de Winter \& Oxnard 2001; Barton \& Harvey 2000) for primate brain evolution. Life-history characteristics can impose considerable constraint on the timing and flexibility of developmental processes and are crucial in determining the potential adaptive pathways available to a population. External mortality drives the onset of reproduction and constrains the investment individuals can make in non- 
reproductive age classes (Charnov 2001). Only longlived individuals that experience low mortality rates can afford the growth and learning period necessary to develop large brains (Clutton-Brock \& Harvey 1980). Similarly, ecological flexibility and complexity have previously been linked to cognitive capacity and brain size in primates (Reader \& Laland 2002; Lefebvre et al. 2004), other mammals (Gittleman 1986) and birds (Lefebvre et al. 1997; Sol et al. 2002, 2005).

However, the associations that have been identified between these traits and brain size have not yet been placed into an overarching framework of brain evolution in primates. Nor has there been any serious attempt to assess how they might relate to the social brain hypothesis. To explore this in more detail, we carried out an evaluation of the ecological and social characteristics associated with primate brain size and social complexity using a three-step analysis. First, we use univariate analyses to determine how different behavioural and ecological traits are associated with measures of relative brain size. Second, we use general linear models to test between alternative models of how these characteristics relate to both brain size and social complexity. Third, we build and test a path model that integrates life history, ecology and social complexity as causes and consequences of brain size. Finally, in addition, we consider the question of the appropriate level of analysis for the brain by comparing analyses using different indices of brain volumetric change, including the brain as a whole, neocortex volume (adjusted in three different ways for scaling effects of body/brain size) and relative cerebellum size. We use cerebellum size as a way of testing whether the functional relationships we describe are generic to all brain components (i.e. simply reflect overall changes in brain volume) or are specific to certain brain units (specifically, the neocortex). Methodological details are given in full in the electronic supplementary material.

\section{(a) Univariate relationships}

We initially explored the relationship between different estimates of relative brain or neocortex size and behavioural, life-history and ecological characteristics using simple univariate tests (either one-way ANOVAs or linear regression).

With the exception of dispersal, all of the indices of sociality were significantly related to indices of brain volume (table 1). However, for each of these behavioural indices, one of the measures of relative neocortex size explained more variation than either total brain size or relative cerebellum size. Indeed, relative cerebellum size was not consistently associated with the social indices. Life-history measures were also associated consistently with the various indices of brain volume. However, in contrast to the social variables, total brain size explained more of the variation than the neocortex size for two of the four life-history variables, basal metabolic rate (BMR) and longevity. Species with large brains have, on average, higher metabolic rates (when corrected for body size), larger bodies, longer life spans and longer juvenile periods. Note, however, that while total brain size is strongly correlated with residual BMR (when neocortex volume and all other variables are partialled out), neocortex volume itself is not correlated with residual BMR when total brain volume is partialled out (figure 1). This relationship implies that life-history traits can permit species to support larger brains (metabolically and developmentally), but the overall architecture of the brain is not itself tied to-or necessarily constrained by-life-history characteristics. Interestingly, the direction of the relationship (or slope) between relative cerebellum size and life history was opposite that of the relationships with brain and neocortex. Overall, the consistent relationship between 'slow' life-history characteristics and higher than predicted metabolic rates indicates that a suite of life-history characteristics are necessary to support the development of large brains. The importance of life history is given additional emphasis by an earlier finding by Joffe (1997) showing that, while total brain size correlates with the conventional gestation + lactation measure of parental investment, relative neocortex volume (at least when the primary visual area is excluded) correlates better with the length of the juvenile period (i.e. weaning to first reproduction), suggesting an important role for social learning of socio-cognitive skills. Thus, extended life histories may be necessary both to allow the laying down of large quantities of brain tissue and to allow that neural tissue to be tuned through social and other more conventional learning processes.

The ecological traits we considered are also consistently related to brain size. The fact that diet is associated with overall brain size, but not with the size of the neocortex relative to the rest of brain, indicates that diet may be a metabolic constraint rather than being cognitively demanding. If diet was cognitively challenging, we could expect that diet would be more strongly associated with executive brain components (e.g. neocortex) rather than total brain size. As with the life-history traits, the relationship between ecological traits and cerebellum size is in the opposite direction to those for total brain and neocortex size. However, the relationships between ecological variables and the various indices of brain size may be an artefact of the fact that both ecology and brain size are strongly associated with group size. Thus, in order to support the energetic needs of all group members, individuals must solve ecological problems in order to maintain large groups. Hence, the association between indices of brain size and ecological characteristics may be causally indirect; it is the behavioural flexibility required to feed group members that drives cognitive evolution rather than the ecological problem solving per se. Alternatively, direct causal relationships between group size and ecology may mean that ecology also covaries with brain size (assuming that brain size is simply a function of group size).

\section{(b) Minimum adequate models}

In order to tease apart the relationship between ecology, group size and brain size, we used forward and backward stepwise general linear models. We constructed a global model of how life history, ecology, brain size and sociality are interrelated using general linear models to identify which sets of characteristics are most strongly associated with brain size, group size and the ecological variables. For this set of analyses, we restrict our brain size estimates to two measurements: 
Table 1. Univiariate relationships between behavioural, ecological and life-history characteristics and relative brain size.

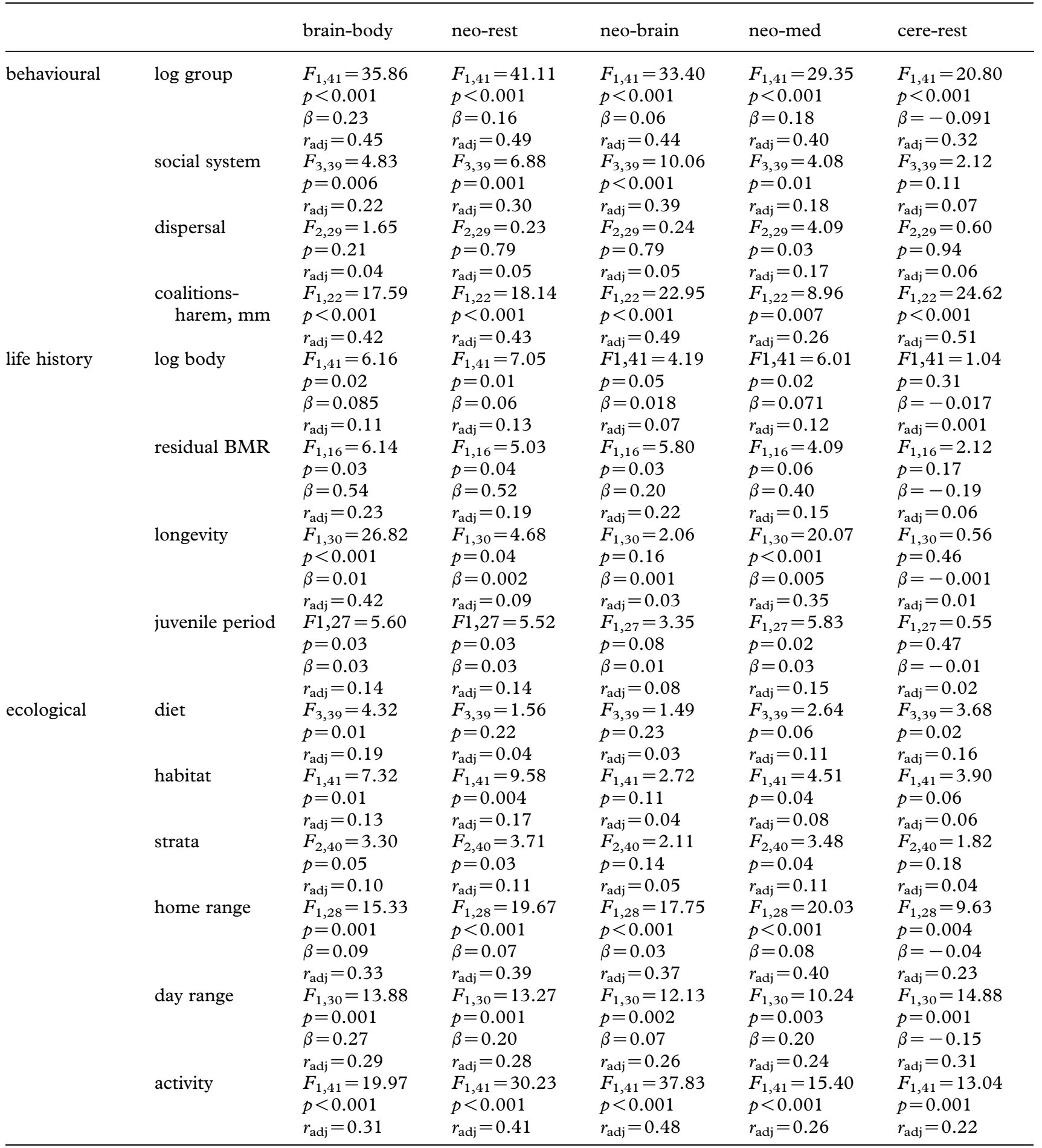

brain/body residuals and neocortex/rest-of-brain residuals. A minimum adequate model (MAM) approach was chosen over an information criterion one because the sample sizes for different parameters are not equal. We used a subset of species with data available for all parameters (see $\$ 3 c$ below) to test whether there was significant phylogenetic autocorrelation in the modelled data by estimating Pagel's $\lambda$ and comparing log-likelihoods of phylogenetic models with those of non-phylogenetic models (Freckleton et al. 2002).

The identified MAM's are summarized in table 2 . Brain size was best explained by a combination of neocortex size and longevity; neocortex size was best explained by total brain size, group size and longevity; home range by body size, group size and day range; and day range by diet and home range. The least stable model was for group size: in this case, three combinations of factors provided equal support (neocortex size and home range; home range and activity; and neocortex size and activity). These models were indistinguishable on the basis of their respective information criteria values. We interpret this as reflecting the complex interaction between ecological (i.e. time budget) and cognitive constraints on group size as previously proposed by Dunbar's (1996, 2002) linear programming model of primate group sizes. 


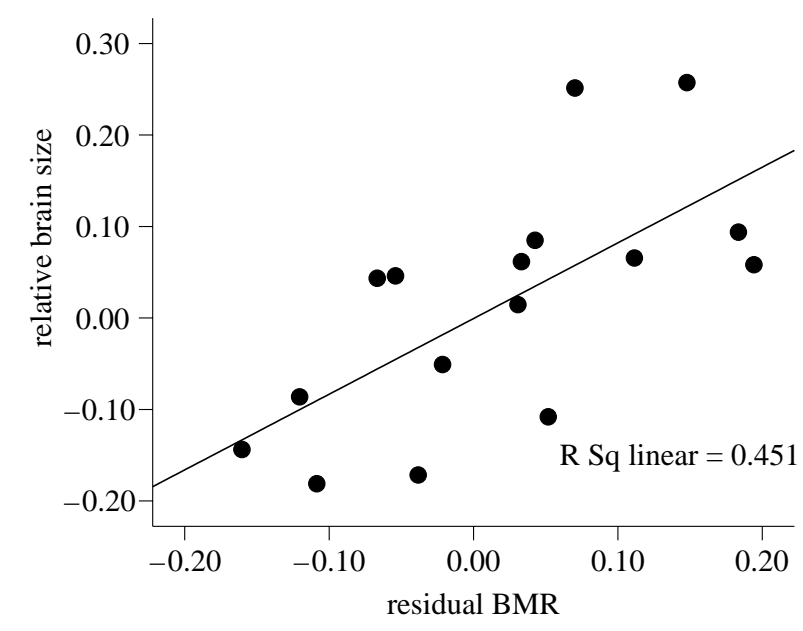

Figure 1. Basal metabolic rate (BMR), controlling for body size (residuals from a linear regression of basal metabolic rate versus log-transformed body size), plotted against relative brain size. Species with higher metabolic rates than expected for their body size also have larger than expected brain size.

We excluded BMR from these analyses, as the sample is too small to provide enough power for discrimination. However, we can use the reduced dataset to identify which factors are associated with BMR and residual BMR. Overall, BMR is most strongly associated with $\operatorname{diet}\left(F_{3,15}=14.85, p<0.001, r_{\text {adj }}^{2}=0.62\right)$; in contrast, residual BMR (or BMR corrected for body size) is strongly associated with both total brain and neocortex size (table 1, figure 1). However, a stepwise regression indicates that residual brain size is the only factor that influences residual BMR (partial $r=0.631, t_{15}=3.15$, $p=0.007$ ); relative neocortex size is dropped from the model (partial $r=0.036, p=0.896$ ). We interpret these results as implying two important conclusions. First, for a given body size, individuals can only invest extra available energy into evolving and maintaining expensive brain architecture if they can commandeer sufficient resources to exceed their basic metabolic requirements. Second, while BMR constrains total brain size, it does not have a strong influence on the way gross brain volume is allocated to different brain units (i.e. on brain architecture).

\section{(c) Path analysis}

We now evaluate how the suite of ecological, lifehistory and social characteristics can be integrated into a global model of primate brain evolution. The model we propose is premised on the following assumptions: (i) inherent life-history characteristics are necessary to allow species to support the development and maintenance of large brains, (ii) these characteristics and the high metabolic demands of large brains drive and/or constrain ecology, and (iii) social complexity (or group size) represents the functional benefit of maintaining large brains. Part of the purpose in building the model is to test of the validity of these assumptions by asking whether they provide us with a better understanding of primate brain evolution than do alternative explanations.

Using the MAM analyses, we integrated the various relationships between life history, sociality, brain size and

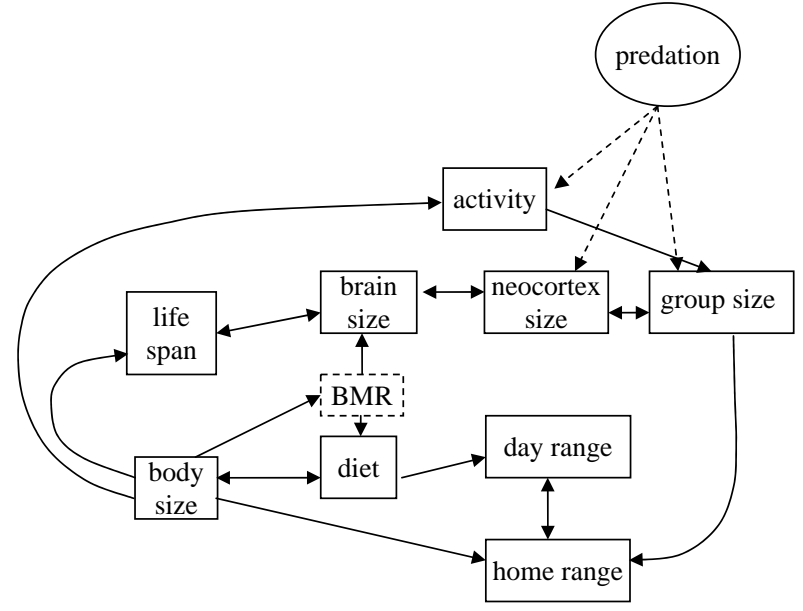

Figure 2. Path diagram of predicted relationship between life history, ecology, brain size and group size in primates. Group size is pushed upwards by external factors such as predation, but is limited by ecological and cognitive constraints. Brain size is, in turn, limited by energetic and life-history constraints.

ecology into a global model of brain evolution and group size (figure 2). We have also used evidence from previous studies to incorporate additional relationships into the path diagram in order to help infer causality. These include BMR correlating with brain size, longevity and life history (Armstrong 1985; Ross 1992; Allman et al. 1993), group size correlating with home range size, day range and predation (Grant et al. 1992; Wrangham et al. 1993; Hill \& Dunbar 1998; Hill \& Lee 1998; Shultz et al. 2004) and, of course, brain size correlating with group size (Dunbar 1992; Barton 1996).

In order to determine whether our proposed model actually provides the best causal explanation for group size, we compared alternative 'causal' models, or pathways, for group size. Our test model (that shown in figure 2) was that the factors most closely associated with group size on the diagram (neocortex, activity and home range size) are better predictors than the factors that are more deeply embedded in the diagram. Although we could potentially test every permutation of factors to identify the 'best of all possible models', in the interests of parsimony we based our candidate alternative models on the MAMs identified in the previous section (table 2). More explicitly, we took group size as the dependent variable and tested a subset of models that focused, in turn, on successive layers of independent variables in the path diagram. This allows us to ask whether our model (which assumes that the three most proximate factors in the path model are the best predictors) is better than a model that considers successively more remote sets of independent variables that, in the model shown in figure 2, only influence group size indirectly. We consider two successively remote layers in the model shown in figure 2: a set of variables that are one step removed from the core variables of our proposed model (e.g. day range, diet, brain size) and the most distal set (body size, diet and lifespan).

We used two methods to compare between alternative models on a complete subset of species with data available for all parameters: (i) change in Bayesian information 
Table 2. Generalized linear matrix MAM results for relationships suggested by the path diagram shown in figure 2 . ( $\lambda$ represents the optimized degree of phylogenetic autocorrelation (or contribution of the species relatedness covariance matrix to the overall model fit), where $\lambda=0$ indicates no autocorrelation and $\lambda=1$ means the degree of covariance between species conforms to the assumption of Brownian motion trait evolution. Parentheses represent whether the log-likelihood of the phylogenetic model varies significantly from a model that does not include phylogeny.)

\begin{tabular}{|c|c|c|c|c|c|c|c|}
\hline factor & $\begin{array}{l}\text { predictors } \\
\text { included }\end{array}$ & model $r_{\text {adj }}^{2}$ & d.f. & $F$ & $p$ & $\lambda$ & factors excluded \\
\hline \multirow[t]{3}{*}{ relative brain size } & max. lifespan & 0.81 & 1,32 & 44.65 & $<0.001$ & 0 & body size, group size, activity, \\
\hline & diet & & 2,32 & 5.83 & 0.003 & (n.s.) & home range size, day range \\
\hline & neocortex & & 1,32 & 47.05 & $<0.001$ & & length, habitat \\
\hline \multirow{3}{*}{$\begin{array}{l}\text { relative neo- } \\
\text { cortex to rest } \\
\text { of brain }\end{array}$} & group size & 0.67 & & 10.23 & 0.003 & & day range, home range, body size, \\
\hline & brain size & & & 24.43 & $<0.001$ & (n.s.) & diet, activity, habitat \\
\hline & max. lifespan & & & 8.49 & 0.006 & & \\
\hline \multirow[t]{6}{*}{ group size } & activity & 0.60 & & 12.08 & 0.001 & 0.13 & diet, body size, lifespan, brain size, \\
\hline & $\begin{array}{l}\text { neocortex } \\
\text { or }\end{array}$ & & & 10.36 & 0.003 & (n.s.) & day range, habitat \\
\hline & home range & & & & & & \\
\hline & activity & & & & & & \\
\hline & & & & & & & \\
\hline & $\begin{array}{l}\text { home range } \\
\text { neocortex }\end{array}$ & & & & & & \\
\hline \multirow[t]{3}{*}{ home range } & body size & 0.76 & & 11.84 & 0.002 & & diet, activity, brain size, \\
\hline & group size & & & 7.58 & 0.01 & (n.s.) & neocortex size, lifespan, habitat \\
\hline & day range & & & 10.93 & 0.003 & & \\
\hline \multirow[t]{2}{*}{ day range } & home range & 0.49 & & 12.94 & 0.002 & & body size, brain size, \\
\hline & diet & & & 3.71 & 0.03 & (n.s.) & $\begin{array}{l}\text { neocortex size, activity, lifespan, } \\
\text { habitat }\end{array}$ \\
\hline
\end{tabular}

Table 3. Information criteria model selection. ( $\chi^{2} \log$-likelihood (LL) and $p$ values represent the $\chi^{2}$ value for the change in loglikelihood values between the best model (1) and other candidate models.)

\begin{tabular}{llllllr}
\hline model & variables & $n$ & BIC & $\Delta$ BIC & $\chi^{2}$ LL & $p$ \\
\hline 1 & neocortex, home range, activity & 24 & 18.6 & 0 & & \\
2 & brain, home range, activity & 24 & 20.63 & 2.03 & 4 & 0.05 \\
3 & brain, day range, activity & 24 & 25.5 & 6.9 & 13.72 & $<0.001$ \\
4 & brain, diet, activity & 24 & 28.93 & 10.33 & 20.58 & $<0.001$ \\
5 & body, diet, lifespan & 24 & 31.3 & 12.7 & 29.54 & $<0.001$ \\
\hline
\end{tabular}

criterion (BIC) values for small sample sizes (Schwartz 1978) and (ii) likelihood ratio test (LRT; Burnham \& Anderson 2002) using the following formula:

LRT $=-2\left(\log \left(L_{1}\right)-\log \left(L_{2}\right)\right)$,

which approximates a $\chi^{2}$-distribution.

The model proposed by the path diagram received strong support over other candidate models: not only does our main model have a lower BIC value than all alternatives, but also all alternative models are significantly less good at predicting group size (table 3 ). This suggests that, of the variables presented in the path diagram, the factors that are most strongly associated with group size are relative neocortex size, activity pattern and home range size. The ecological and life-history variables that are embedded in the diagram help to facilitate (or, alternatively, constrain) the maintenance of large brains and thus large group size, but are not directly causally related to the relationship between neocortex size and group size.

We interpret external factors such as predation as having a ratchet effect on the system, putting upward pressure on group size, and thus in turn brain size and, through that, ultimately life history. That predation risk is a critical factor influencing primate brain evolution is indicated by two different analyses. First, Shultz et al. (2004) showed that gross predation rate (summed predation rates from all predators on an individual prey species) is a negative function of social group size. Second, Shultz \& Dunbar (2007) have shown that predator bias (the relative rate with which a predator takes a prey species' abundance in the ecological community) is a negative function of brain size (relative to body size), and that this relationship is consistent across two different predators (chimpanzees and large felids) at six different sites on two continents. In other words, predation seems to be imposing both direct selection on brain size (acting through prey species' ability to evade predation attempts, whether this is done at an individual or a social level) and indirect selection via the buffering effect of large group size (with large group size in turn selecting for large brain size). The importance of predation, combined with the lack of any direct role for diet, leads us to conclude that the demands of foraging per se have not been the main driving force of primate cognitive/brain evolution, although foraging innovation may well have been a beneficial by-product of the cognitive sophistication required by social strategies designed to minimize predation risk. 
The path diagram and analyses indicate that there are two pathways that limit maximum group size: one cognitive and one ecological. Because residual BMR explains much more of the variance in brain size than diet does, we can infer that a high energy diet is necessary to support a large brain, but that a large brain is not vital for managing a high quality diet. Large brained species must be able to support their brains metabolically, and this constrains the range of possible diets and energy use patterns to those that provide sufficient surplus calories. Operating from the other end, individuals in large groups have to be able to mitigate the heightened competition for resources between group members. For species with limiting resources, this means expanding home ranges or, in those with contestable resources, day range length (Isbell 1991). The intersection of these two limiting factors can be used to describe the maximum group size obtainable by any population (Dunbar 1996). Once group size is pushed past either the ecological or cognitive limits of the species, groups are expected to either fission or adapt a fission-fusion social structure (Dunbar 1996).

\section{THE NATURE OF SOCIAL COMPLEXITY}

Although the social brain hypothesis has often been formulated in terms of group size, it is more correct to think of it in terms of the complexity of social relationships. Several studies have now produced evidence to support this claim. Kudo \& Dunbar (2001), for example, showed that the size of grooming cliques (interpreted as coalitions) correlated significantly with neocortex ratio (neocortex volume divided by the volume of the rest of the brain) across primates, while Byrne \& Corp (2004) showed that frequencies of tactical deception (standardized for the frequencies with which species have been studied) also correlate with neocortex ratio. The latter finding is of particular interest for the fact that it focuses on cognitively complex behaviour (tactical deception, whereby animals appear to deliberately mislead other individuals). Similarly, Pawlowski et al. (1998) showed that, when number of males in the group is partialled out, neocortex ratio negatively predicts the correlation between male dominance rank and mating success. In effect, low-ranking males in large-brained species do not simply accept a poor return in terms of mating rate, but rather exploit subtle social strategies like alliances or female choice to circumvent what would otherwise be the high-rank males' power-based monopoly over matings. Similarly, when we compared relative neocortex volume in species that habitually form coalitions with those that do not (as defined by Plavcan et al. 1995), we found that species in which coalitions are reported to be common have significantly larger neocortices, when all other variables including phylogeny are held constant (figure 3). Finally, Lewis (2000) has shown, for a small sample of primate species, that the proportion of all play that is social (as opposed to solitary or instrumental) is also positively correlated with neocortex ratio.

The latter finding highlights another frequently overlooked aspect of cognitive evolution, namely an important role for development and learning by experience in a social context. Having a large brain

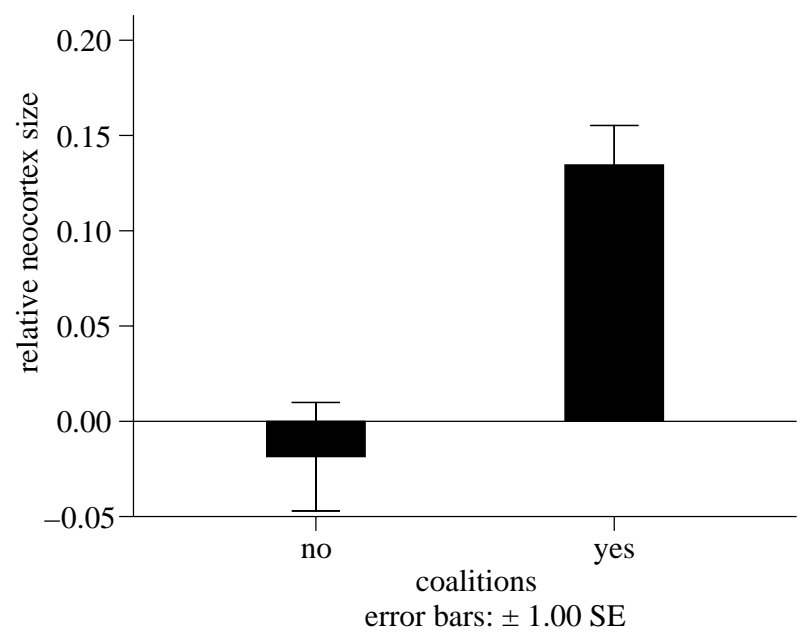

Figure 3. Relative neocortex size (as measured by a linear regression of log-transformed neocortex volume over logtransformed volume of the rest of the brain) in species that do and do not form coalitions with other group members (solitary and monogamous species excluded from analysis). We use Plavcan et al.'s (1995) categorization of coalitionforming species.

may not, of itself, be sufficient to allow an animal to engage in complex social behaviour; the brain wetware merely provides the capacity. The hardware needs the equivalent of software programming and this comes through the learning experiences of socialization. Social play offers one context in which that learning takes place. In support of this, Joffe (1997) showed that the volume of the neocortex frontal to the primary visual area is better predicted by the length of the period of socialization (the period between weaning and first reproduction). Growing up in a social environment may thus be at least as important as having a brain of the right size.

It is worth noting here that these analyses do not all use the Stephan brain database. Several of the analyses reported in this section used neocortex ratios estimated from cranial volume because some of the species in an analysis did not occur in the Stephan database. Indeed, some of the analyses reported here have used databases obtained by different methods using different specimens (e.g. Fuster's prefrontal cortex dataset, Semedenferi's frontal lobe dataset and Rilling's magnetic resonance imaging-derived brain component volume dataset), with only partial overlap in the species contained in each database. Despite this, the results are robustly consistent. We make this point because over-reliance of the Stephan database has sometimes been raised as a criticism.

\section{HOW TO EVOLVE A BIG BRAIN}

This leads us to the final issue, namely the question of how brain evolution has occurred. There has been considerable debate in the recent literature as to whether developmental constraints have forced a significant degree of uniformity on brain structure, i.e. species differences in the volume of particular brain units merely reflect species differences in total brain volume (Finlay \& Darlington 1995; Finlay et al. 2001; de Winter \& Oxnard 2001), or whether there has been mosaic evolution whereby some brain units have 
enlarged more rapidly than others (Barton \& Harvey 2000). There can be no doubt about the fact that there must be both developmental (Martin 1981) and energetic (Aiello \& Wheeler 1995) constraints on final brain size; indeed, we have demonstrated above that such constraints do exist. However, the real issue here is whether brain units enlarge proportionally as total brain volume enlarges (Finlay et al. 2001), or do so disproportionately as a function of specific selection pressures (Barton \& Harvey 2000). There is currently no real agreement on this.

One reason why there may be proportional convergence in brain component volumes is that higher order representations of sensory systems within the brain seem to be organized on a direct functional basis: upstream systems seem to be volumetrically correlated with their input systems (Stevens 2001). Thus, increasing convergence in proportional volumes may reflect increasing integration of units from different functional groupings as a result of increased sharing of information. In support of this it is known that, in the primate brain, there are direct axonal links from subcortical areas like the amygdala and the cerebellum to the frontal lobe of the neocortex, whereas this is much less extensively the case in carnivores (Fuster 1988). Using a different approach, we were here able to confirm that the cerebellum, at least, is unrelated to social group size when total brain and neocortex volumes are partialled out. Interestingly, cerebellum volume correlates negatively with those life-history variables that correlate positively with neocortex volume, suggesting that not all brain units are under the same linked selection regime (see also Barton \& Harvey 2000).

A more general issue of some importance concerns the overall pattern of brain evolution. Finlay \& Darlington (1995) have pointed out that, although brain units scale very tightly with each other, the scaling coefficient is not always unity. Of particular significance in the present context is the fact that, across mammals, the scaling coefficient for neocortex volume relative to the brain as a whole is significantly higher than unity: in fact, the scaling relationship against the whole brain ranges from 1.103 for neocortex as a whole, to 1.115 for the frontal lobe (Semendeferi et al. 1997), indicating that the neocortex in general, and the frontal lobe in particular, have increased disproportionately during the course of primate brain evolution. Largebrained primates like apes and humans have disproportionately large frontal lobes-even though, as Semendeferi et al. (1997) pointed out, they do not deviate from the general primate allometric scaling relationship. This is significant because the frontal lobe is widely understood to be primarily responsible both for integration across sensory and association units and for those cognitive processes generally referred to as 'executive functions' (Kolb \& Wishaw 1996).

One reason for the steep positive allometric scaling of the frontal lobe is the fact that the brain evolves (and, indeed, develops and myelinates; Gogtay et al. 2004) from back (the visual areas) to front (the executive areas). Thus, when brain evolution occurs, it mainly involves adding more frontal cortex rather than increasing all brain units proportionately. Given that visual acuity is limited only by retinal area and not by

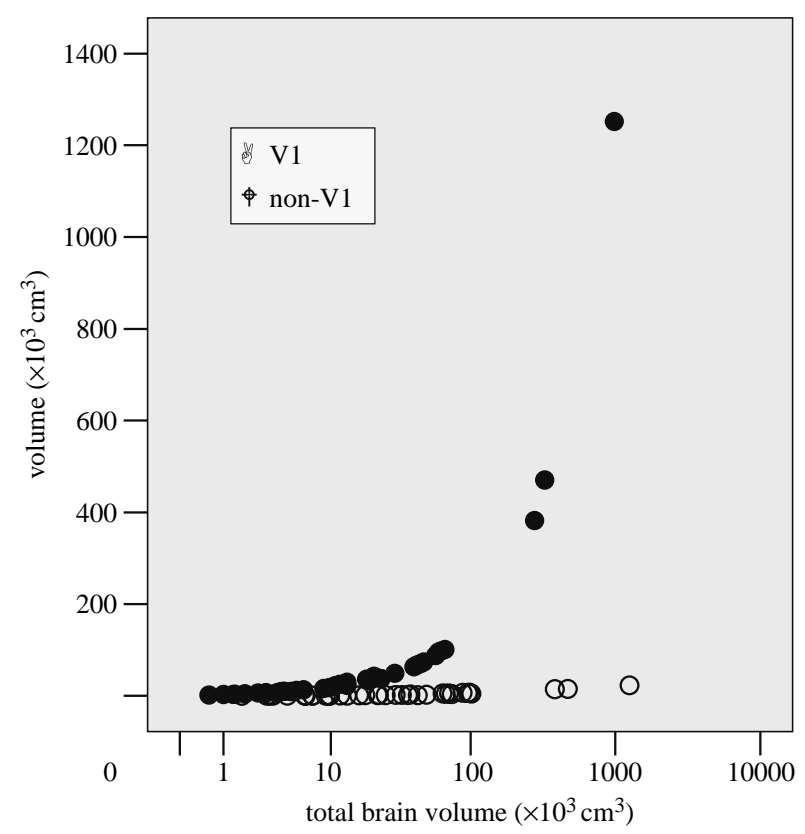

Figure 4. Volumes of primary visual cortex (V1) and rest of neocortex (non-V1) plotted against total brain volume for primates. Source: data from Stephan et al. (1981).

body size or total brain size, there is limited value in adding more visual cortex (located mainly in the occipital lobe at the back of the brain) than is minimally necessary to map the inputs from the retina. This effectively means that there is increasing frontal lobe volume available for executive-type functions. Dunbar (2003) showed that, in primates, the area of the primary visual cortex (commonly known as V1) is linearly (and tightly) related to the both volume of the visual pathway (lateral geniculate nucleus and the visual tract) and the volume of the orbit (the main factor determining retinal area). Figure 4 shows the consequences of this; the volume of the primary visual cortex (V1) quickly reaches an asymptotic value as a function of total brain volume, but the volume of the rest of the neocortex (non-V1 cortex) increases dramatically. Unfortunately, with Stephan's database, we are unable to partition the neocortex down into smaller units, but the volumetric analyses provided by Semendeferi et al. (1997) for frontal lobe and by Fuster (1988) for prefrontal cortex imply even steeper relationships for these more frontal units that are specifically involved in cognitive executive functions. Since it is these rather than the visual areas per se that are likely to be responsible for the social brain effect, it may be no accident that increasing brain size correlates with increasing social skills (and hence group size).

\section{CONCLUSIONS}

We have shown that brain volume (and in primates, specifically neocortex volume) correlates with sociality. In primates, this emerges as a strong relationship with social group size, but this belies a deeper relationship with behavioural indices of social complexity (including coalition formation, the use of tactical deception, the use of more subtle social strategies, and social play). More intriguingly, however, there is some evidence to suggest that there may be a phase shift in the form of the 
social brain between primates (where the social brain hypothesis has a quantitative form: a direct correlation with social group size) and non-primate mammals (where it has a qualitative form: a correlation with sociality, but not social group size). At present, we do not understand why this should be so, but it may suggest one reason why primates seem to be in a different social league to non-primates. We have yet to explore whether, within this general pattern, some non-primate taxa exhibit any kind of convergence in this respect (elephants and toothed whales are obvious candidates).

A further important issue is the fact that brain evolution involves a suite of traits (an adaptive complex): constraints or consequences arise in the context of life history, development, diet and other aspects of ecology as well as behaviour, and these have to be solved if a species is to be able to increase its brain (or neocortex) size. Since neocortex size constrains group size, major phase shifts in constraint variables may be necessary for a species to increase its group size in response to an ecological challenge.

It is important to remember that primates, in particular, may be under a dual constraint on group size. Not only may the cohesion of groups be limited by their cognitive abilities, but they may also be constrained by time budgeting issues that arise from ecology (Dunbar 1996; Lehmann et al. in press). Animals have to invest time in grooming in order to create social bonds of sufficient intensity to enable large groups of individuals to forage together as a cohesive social unit. If the size of foraging groups demanded by the ecology exceeds those with which the species can cope on either or both of these dimensions, it may not necessarily be impossible for the species to live in groups of this size, but there will be dramatic consequences for their social cohesiveness. We would predict a much looser form of sociality and a greater degree of fission-fusion in these cases.

We have identified the neocortex as critical in this context (at least within primates), and the evidence points to the particular importance of the more frontal units (especially the frontal lobe). However, at present we have very little idea as to how the brain produces these effects. We have suggested that, as brain size increases, a disproportionate amount of executive function computational power becomes available, allowing for increasingly sophisticated social behaviour. However, our understanding of the mechanisms involved in how the brain handles any kind of secondary information processing (i.e. anything above basic sensory processing) is very limited at present. Nor do we have much idea as to the genetic mechanisms involved, although recent studies have indicated the existence of genes that play an important role in the neurophysiology of brain activity, e.g. clearing neurons of the by-products of activity, so as to reduce their refractory times (Dorus et al. 2004).

\section{REFERENCES}

Aiello, L. C. \& Wheeler, P. T. 1995 The expensive tissue hypothesis: the brain and the digestive system in human evolution. Curr. Anthropol. 36, 199-221. (doi:10.1086/ 204350)
Allman, J., McLaughlin, T. \& Hakeem, A. 1993 Brain-weight and life-span in primate species. Proc. Natl Acad. Sci. USA 90, 118-122. (doi:10.1073/pnas.90.1.118)

Armstrong, E. 1985 Relative brain size in monkeys and prosimians. Am. F. Phys. Anthropol. 66, 263-273. (doi:10. 1002/ajpa.1330660303)

Barton, R. A. 1993 Independent contrasts analysis of neocortex size and socioecology in primates. Behav. Brain Sci. 16, 694-695.

Barton, R. A. 1996 Neocortex size and behavioural ecology in primates. Proc. R. Soc. B 263, 173-177. (doi:10.1098/ rspb.1996.0028)

Barton, R. A. \& Dunbar, R. I. M. 1997 Evolution of the social brain. In Machiavellian intelligence II (eds A. Whiten \& R. W. Byrne), pp. 240-263. Cambridge, UK: Cambridge University Press.

Barton, R. A. \& Harvey, P. H. 2000 Mosaic evolution of brain structure in mammals. Nature 405, 1055-1058. (doi:10. 1038/35016580)

Burnham, K. P. \& Anderson, D. R. 2002 Model selection and multi-model inference: a practical information-theoretic approach. New York, NY: Springer.

Byrne, R. W. \& Corp, N. 2004 Neocortex size predicts deception rate in primates. Proc. R. Soc. B 271, 1693-1699. (doi:10.1098/rspb.2004.2780)

Byrne, R. W. \& Whiten, A. 1988 Machiavellian intelligence: social expertise and the evolution of intelligence in monkeys, apes and humans. Oxford, UK: Oxford University Press.

Charnov, E. L. 2001 Evolution of mammal life histories. Evol. Ecol. Res. 3, 521-535.

Clutton-Brock, T. H. \& Harvey, P. H. 1980 Primates, brains and ecology. F. Zool. 190, 309-323.

Deaner, R. O., Nunn, C. L. \& van Schaik, C. P. 2000 Comparative tests of primate cognition: different scaling methods produce different results. Brain Behav. Evol. 55, 44-52. (doi:10.1159/000006641)

Deaner, R. O., Barton, R. A. \& van Schaik, C. P. 2003 Primate brains and life histories: renewing the connection. In Primate life histories and socioecology (eds P. M. Kappeler \& M. E. Pereira), pp. 233-265. Cambridge, UK: Cambridge University Press.

Dorus, S., Vallender, E. J., Evans, P. D., Anderson, J. R., Gilbert, S. L., Mahowald, M., Wyckoff, G. J., Malcom, C. M. \& Lahn, B. T. 2004 Accelerated evolution of nervous system genes in the origin of Homo sapiens. Cell 119, 1027-1040. (doi:10.1016/j.cell.2004.11.040)

Dunbar, R. I. M. 1988 Primate social systems. London, UK: Chapman and Hall.

Dunbar, R. I. M. 1992 Neocortex size as a constraint on group-size in primates. F. Hum. Evol. 22, 469-493. (doi:10.1016/0047-2484(92)90081-J)

Dunbar, R. I. M. 1996 Determinants of group size in primates: a general model. In Evolution of culture and language in primates and humans (eds J. Maynard Smith, G. Runciman \& R. Dunbar), pp. 33-57. Oxford, UK: Oxford University Press.

Dunbar, R. I. M. 1998 The social brain hypothesis. Evol. Anthropol. 6, 178-190. (doi:10.1002/(SICI)1520-6505 (1998)6:5<178::AID-EVAN5>3.0.CO;2-8)

Dunbar, R. I. M. 2002 Modelling primate behavioural ecology. Int. f. Primatol. 23, 785-819. (doi:10.1023/ A:1015576915296)

Dunbar, R. I. M. 2003 Why are apes so smart? In Primate life histories and socioecology (eds P. Kappeler \& M. Pereira), pp. 285-298. Chicago, IL: Chicago University Press.

Dunbar, R. I. M. \& Bever, J. 1998 Neocortex size predicts group size in carnivores and some insectivores. Ethology 104, 695-708. 
Finlay, B. L. \& Darlington, R. B. 1995 Linked regularities in the development and evolution of mammalian brains. Science 268, 1578-1584. (doi:10.1126/science.7777856)

Finlay, B. L., Darlington, R. B. \& Nicastro, N. 2001 Developmental structure in brain evolution. Behav. Brain Sci. 24, 263-308. (doi:10.1017/S0140525X01003958)

Freckleton, R. P., Harvey, P. H. \& Pagel, M. 2002 Phylogenetic analysis and comparative data: a test and review of evidence. Am. Nat. 160, 712-726. (doi:10.1086/343873)

Fuster, J. M. 1988 The prefrontal cortex: anatomy, physiology and neuropsychology of the frontal lobe. New York, NY: Lippincott Williams \& Wilkins.

Gittleman, J. L. 1986 Carnivore brain size, behavioral ecology, and phylogeny. F. Mammal. 67, 23-36. (doi:10. 2307/1380998)

Gogtay, N. et al. 2004 Dynamic mapping of human cortical development during childhood through early adulthood. Proc. Natl Acad. Sci. USA 101, 8174-8179. (doi:10. 1073/pnas.0402680101)

Grant, J. W. A., Chapman, C. A. \& Richardson, K. S. 1992 Defended versus undefended home range size of carnivores, ungulates and primates. Behav. Ecol. Sociobiol. 31, 149-161. (doi:10.1007/BF00168642)

Hill, R. A. \& Dunbar, R. I. M. 1998 An evaluation of the roles of predation rate and predation risk as selective pressures on primate grouping behaviour. Behaviour 135, 411-430.

Hill, R. A. \& Lee, P. C. 1998 Predation risk as an influence on group size in cercopithecoid primates: implications for social structure. F. Zool. 245, 447-456. (doi:10.1111/ j.1469-7998.1998.tb00119.x)

Isbell, L. A. 1991 Contest and scramble competition: patterns of female aggression and ranging behaviour among primates. Behav. Ecol. 2, 143-155.

Joffe, T. H. 1997 Social pressures have selected for an extended juvenile period in primates. F. Hum. Evol. 32, 593-605. (doi:10.1006/jhev.1997.0140)

Kolb, B. \& Wishaw, I. Q. 1996 Fundamentals of human neuropsychology. San Francisco, CA: Freeman.

Kudo, H. \& Dunbar, R. I. M. 2001 Neocortex size and social network size in primates. Anim. Behav. 62, 711-722. (doi:10.1006/anbe.2001.1808)

Lefebvre, L., Whittle, P., Lascaris, E. \& Finkelstein, A. 1997 Feeding innovations and forebrain size in birds. Anim. Behav. 53, 549-560. (doi:10.1006/anbe.1996.0330)

Lefebvre, L., Reader, S. M. \& Sol, D. 2004 Brains, innovations and evolution in birds and primates. Brain Behav. Evol. 63, 233-246. (doi:10.1159/000076784)

Lehmann, J., Korstjens, A. H. \& Dunbar, R. I. M. In press. Group size, grooming and social cohesion in primates. Anim. Behav.

Lewis, K. P. 2000 A comparative study of primate play behaviour: implications for the study of cognition. Folia Primatol. 71, 417-421. (doi:10.1159/000052740)

Marino, L. 1996 What can dolphins tell us about primate evolution? Evol. Anthropol. 5, 81-86. (doi:10. 1002/(SICI) 1520-6505(1996)5:3 < 81::AID-EVAN3>3.0. $\mathrm{CO} ; 2-\mathrm{Z})$

Marino, L. 2004 Cetacean brain evolution: multiplication generates complexity. F. Comp. Psychol. 17, 1-16.

Martin, R. D. 1981 Relative brain size and basal metabolicrate in terrestrial vertebrates. Nature 293, 57-60. (doi:10. $1038 / 293057 \mathrm{a} 0$ )
Pawlowski, B., Lowen, C. B. \& Dunbar, R. I. M. 1998 Neocortex size, social skills and mating success in primates. Behaviour 135, 357-368.

Perez-Barberia, F. J. \& Gordon, I. J. 2005 Gregariousness increases brain size in ungulates. Oecologia 145, 41-52.

Perez-Barberia, F. J., Shultz, S. \& Dunbar, R. I. M. Submitted. Evidence for intense coevolution of sociality and brain size in three orders of mammals.

Plavcan, J. M., van Schaik, C. P. \& Kappeler, P. M. 1995 Competition, coalitions and canine size in primates. f. Hum. Evol. 28, 245-276. (doi:10.1006/jhev.1995.1019)

Reader, S. M. \& Laland, K. N. 2002 Social intelligence, innovation, and enhanced brain size in primates. Proc. Natl Acad. Sci. USA 99, 4436-4441. (doi:10.1073/pnas. 062041299)

Ross, C. 1992 Environmental correlates of the intrinsic rate of natural increase in primates. Oecologia 90, 383-390. (doi:10.1007/BF00317695)

Sawaguchi, T. \& Kudo, H. 1990 Neocortical development and social-structure in primates. Primates 31, 283-289. (doi:10.1007/BF02380949)

Schwartz, G. 1978 Estimating the dimension of a model. Ann. Statistics 6, 461-464.

Semendeferi, K., Damasio, H. \& Frank, R. 1997 The evolution of the frontal lobes: a volumetric analysis based on three-dimensional reconstructions of magnetic resonance scans of human and ape brains. F. Hum. Evol. 32, 375-388. (doi:10.1006/jhev.1996.0099)

Shultz, S. \& Dunbar, R. I. M. 2006 Both social and ecological factors predict ungulate brain size. Proc. R. Soc. B 273, 207-215. (doi:10.1098/rspb.2005.3283)

Shultz, S. \& Dunbar, R. I. M. 2007 Chimpanzee and felid diet composition is influenced by prey brain size. Biol. Lett. 2, 505-508. (doi:10.1098/rsbl.2006.0519)

Shultz, S., Noe, R., McGraw, W. S. \& Dunbar, R. I. M. 2004 A community-level evaluation of the impact of prey behavioural and ecological characteristics on predator diet composition. Proc. R. Soc. B 271, 725-732. (doi:10. 1098/rspb.2003.2626)

Sol, D., Timmermans, S. \& Lefebvre, L. 2002 Behavioural flexibility and invasion success in birds. Anim. Behav. 63, 495-502. (doi:10.1006/anbe.2001.1953)

Sol, D., Duncan, R. P., Blackburn, T. M., Cassey, P. \& Lefebvre, L. 2005 Big brains, enhanced cognition, and response of birds to novel environments. Proc. Natl Acad. Sci. USA 102, 5460-5465. (doi:10.1073/pnas.0408145102)

Stephan, H., Frahm, H. \& Baron, G. 1981 New and revised data on volumes of brain structures in insectivores and primates. Folia Primatol. 35, 1-29.

Stevens, C. F. 2001 An evolutionary scaling law for the primate visual system and its basis in cortical function. Nature 411, 193-195. (doi:10.1038/35075572)

de Winter, W. \& Oxnard, C. E. 2001 Evolutionary radiations and convergences in the structural organisation of mammalian brains. Nature 409, 710-714. (doi:10.1038/35055547)

Wrangham, R. W., Gittleman, J. L. \& Chapman, C. A. 1993 Constraints on group-size in primates and carnivorespopulation-density and day-range as assays of exploitation competition. Behav. Ecol. Sociobiol. 32, 199-209. (doi:10. 1007/BF00173778) 